\title{
Successful bortezomib treatment in combination with dexamethasone and thalidomide for previously untreated epidural plasmacytoma
}

\author{
ZHIYONG ZENG* , LING ZHENG* , JUNFANG LIN and JUNMIN CHEN \\ Department of Hematology and Rheumatology, The First Affiliated Hospital \\ of Fujian Medical University, Fuzhou 350005, P.R. China
}

Received September 30, 2011; Accepted December 13, 2011

DOI: $10.3892 / \mathrm{ol} .2011 .539$

\begin{abstract}
Epidural plasmacytoma is a rare clinical entity of plasma cell neoplasms. The optimal management of extramedullary plasmacytoma remains unclear, particularly for bulky masses. In this study, we report the case of a 35-year-old male who presented with the inability to walk, urinary incontinence and superficial and deep sensory disturbance. Spinal magnetic resonance imaging revealed a large epidural mass compressing the spinal cord at the level of T2-T4. The patient underwent surgical T2-T4 vertebral canal decompression. Histopathological examination and other findings confirmed the diagnosis of extramedullary plasmacytoma. The patient was treated with bortezomib in combination with dexamethasone and thalidomide. Following 4 cycles of chemotherapy, the patient achieved an excellent clinical response. Over 1 year following the initial diagnosis, the patient was in complete remission with no evidence of local relapse or evolution to multiple myeloma. This is the first case of a previously untreated epidural plasmacytoma, which was successfully treated with bortezomib-containing chemotherapy.
\end{abstract}

\section{Introduction}

Multiple myeloma(MM) is a malignant neoplasm of plasma cells that accumulate in the bone marrow, and accounts for approximately $10 \%$ of all hematological malignancies (1). Multiple myeloma is characterized by skeletal destruction, renal failure, hypercalcemia and monoclonal immunoglobulin (M protein) accumulation in the serum or urine. The solitary extramedullary plasmacytoma (SEP) is a rare form of tumor, accounting for

Correspondence to: Professor Junmin Chen, Department of Hematology and Rheumatology, The First Affiliated Hospital of Fujian Medical University, Chating, Fuzhou 350005, P.R. China

E-mail: drjunminchen@hotmail.com

*Contributed equally

Key words: bortezomib, epidural, plasmacytoma less than $3 \%$ of all plasma cell neoplasms. SEPs are localized mainly in the the submucosa of various organs in the head and neck; however, epidural space involvement is relatively rare (2). The diagnosis of SEP is based on histological confirmation of a single extramedullary mass of plasma cells with no evidence of multiple myeloma (3). The SEP size has been reported to be a poor prognostic factor (4). Therefore, the treatment of large SEP remains a challenge. In the present study, we report the case of a previously untreated patient with a large epidural plasmacytoma who achieved an excellent clinical response and sustained remission following bortezomib treatment. The study was approved by the ethics committee of Fujian Medical University. Informed consent was obtained from the patient who participated in the study.

\section{Case report}

This report presents the case of a 35-year-old male with no significant clinical background. At the time of referral to The First Affiliated Hospital of Fujian Medical University (China), the patient had suffered from thoracic back pain for 2 months and progressively ascending bilateral numbness and weakness of the lower extremities for 1 month. Shortly following a visit to the neurology clinic, the patient became unable to walk independently and developed urinary incontinence. Neurological examination revealed that muscular strength was normal in the upper limbs, but decreased in the lower limbs (grade $1 / 5$ strength in the left and grade $2 / 5$ in the right). Muscular tension increased in the lower limbs. Superficial and deep sensation disturbance was found below the level of T5 for the left side and T6 for the right side, respectively. The reflexes did not exist in the two sides, including cremaster, patellar tendon and abdominal wall at the upper, middle and lower level. Signs of Babinski, Oppenheim, Chaddock and Gordon were positive in the two sides. Cranial computed tomography (CT) and magnetic resonance imaging (MRI) were normal. Plain MRI of the thoracic spine revealed an epidural mass $(6.4 \times 1.4 \times 4.2 \mathrm{~cm})$, which was located at the T2-T4 level where it compressed the spinal cord. The mass was isointense to the spinal cord on the T1-weighted image (Fig. 1A) and hyperintense on the T2-weighted image (Fig. 1B), with a moderate and homogenous contrast enhancement (Fig. 1C). Bone destruction 

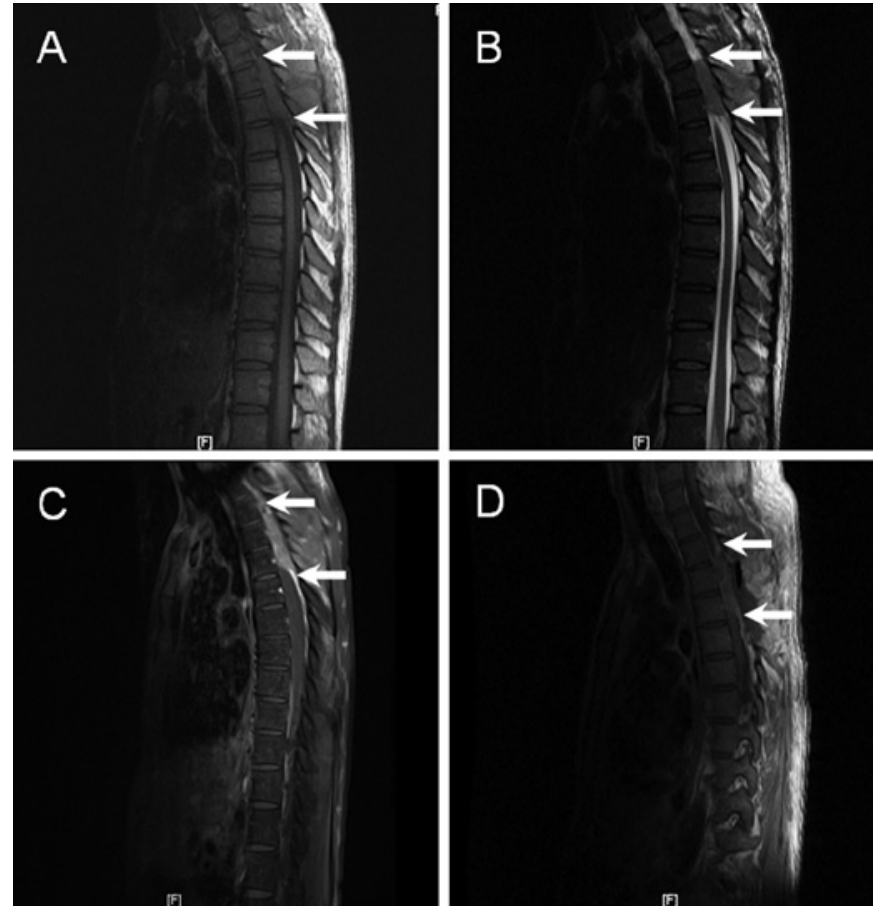

Figure 1. Sagittal MRI of the thoracic spine revealed an epidural mass at the level of T2-T4 where it compressed the spinal cord (arrowheads) (A) T1-weighted image at the onset of disease demonstrating isointensity to the spinal cord; (B) T2-weighted image at the onset of disease demonstrating hyperintensity to the spinal cord; (C) enhanced T1-weighted image at the onset of disease demonstrating a moderate and homogenous contrast enhancement; (D) enhanced T1-weighted image following vertebral canal decompression demonstrating the remaining mass still existed in the same position and compressed the spinal cord. MRI, magnetic resonance imaging.

was observed in the corresponding spinal and vertebral plate. The right lateral neighboring muscles were not well organized.

The patient underwent neurosurgical intervention with T2-T4 vertebral canal decompression and excision of the extradural tumour. However, a postoperative MRI of the thoracic spine revealed that only part of the epidural mass had been removed. Slight contrast enhancement showed that the remaining mass had been maintained in the same position (Fig. 1D). Histopathological analysis of the tumour tissue revealed that the cells expressed CD38 and CD138, which were markers of plasma cells. However, CD20, a typical immunophenotype for B lymphocytes, was negative. The patient was referred to the hematology department for further investigation following the announcement of the histopathology report. At that time, laboratory work-up findings revealed an erythrocyte sedimentation rate (ESR) of $20 \mathrm{~mm} / \mathrm{h}$, C-reactive protein of $5.76 \mathrm{mg} / 1$, white blood cell count of $11.48 \times 10^{9} / 1$, hemoglobin of $129 \mathrm{~g} / 1$ and platelets $175 \times 10^{12} / 1$. The serum renal and liver function tests were within normal ranges. The $\beta_{2}$-microglobulin level was $1.74 \mathrm{mg} / \mathrm{l}$ (reference range: $0.7-1.8 \mathrm{mg} / \mathrm{l})$. Bence-Jones protein was negative. The serum concentrations of immunoglobulins were as follows: IgG $10.6 \mathrm{~g} / 1, \operatorname{IgA} 2.62 \mathrm{~g} / 1, \operatorname{IgM} 0.86 \mathrm{~g} / 1, \kappa 2.5 \mathrm{~g} / 1$ and $\lambda 1.9 \mathrm{~g} / 1$. Serum protein electrophoresis revealed no abnormal spike, and immunofixation electrophoresis revealed polyclonal bands. Bone marrow examination revealed $7.5 \%$ well-differentiated plasma cells. These findings were utilized in making the diagnosis of extraosseous epidural plasmacytoma.
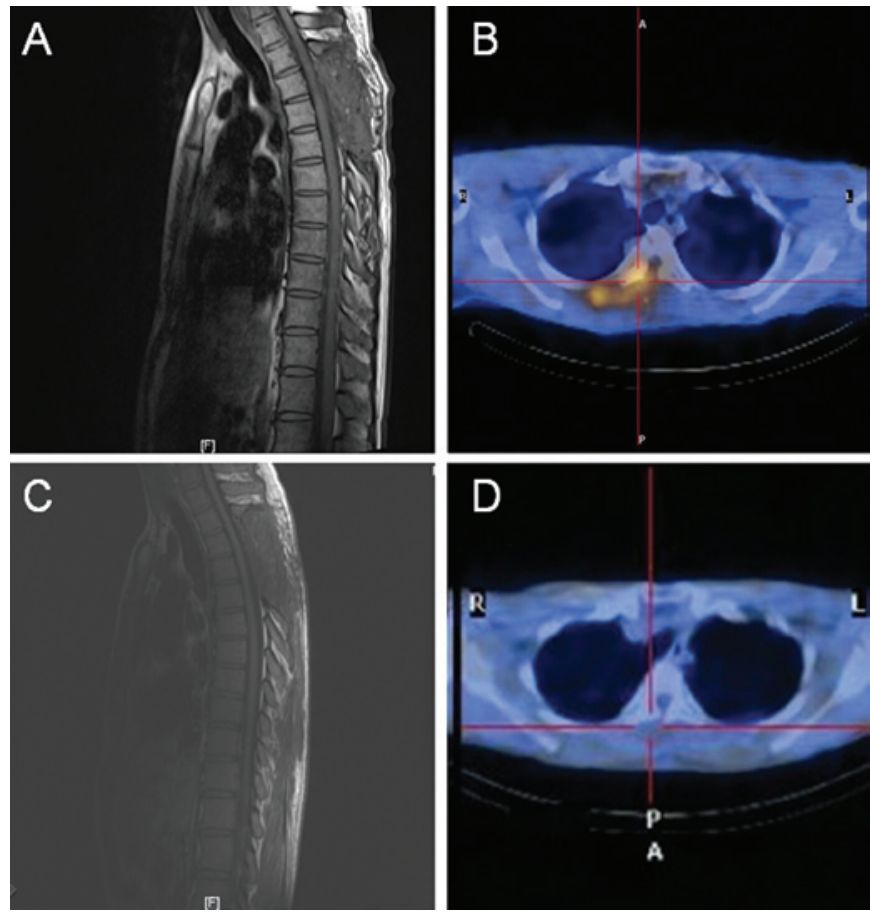

Figure 2. Following the first cycle of chemotherapy, (A) sagittal T1-weighted MRI of the thoracic spine revealed that the epidural mass markedly decreased; however, (B) ${ }^{18} \mathrm{~F}$-FDG PET/CT revealed focal areas of increased ${ }^{18} \mathrm{~F}$-FDG uptake. Over 1 year following the initial diagnosis, (C) sagittal T1-weighted MRI of the thoracic spine revealed no relapse and (D) ${ }^{18} \mathrm{~F}-\mathrm{FDG}$ PET/CT revealed no clear ${ }^{18} \mathrm{~F}-\mathrm{FDG}$ uptake. MRI, magnetic resonance imaging; PET, positron emission tomography; CT, computed tomography; ${ }^{18} \mathrm{~F}-\mathrm{FDG}$, ${ }^{18}$ F-fluorodeoxyglucose.

The patient received bortezomib at a dose of $1.0 \mathrm{mg} / \mathrm{m}^{2}$ on days $1,4,8$ and 11 of a 21-day cycle in combination with a dose of $40 \mathrm{mg}$ dexamethasone intravenously on days 1,2 , $4,5,8,9$ and 11 and 12 of each cycle, as well as a dose of $75 \mathrm{mg}$ thalidomide daily. Following completion of the first cycle of combination chemotherapy, the automatic micturition function returned to normal. Back pain was significantly reduced and the strength in the patient's lower limbs gradually improved. A repeat MRI revealed complete remission of the epidural mass with decompression of the spinal cord (Fig. 2A); however, ${ }^{18} \mathrm{~F}$-fluorodeoxyglucose $\left({ }^{18} \mathrm{~F}\right.$-FDG) positron emission tomography (PET)/CT scan revealed focal areas of increased ${ }^{18}$ F-FDG uptake (Fig. 2B). The patient experienced 4 cycles of chemotherapy, which were well tolerated and no side effects were documented. Over 1 year following the initial diagnosis, there was no evidence of relapse in the repeat MRI (Fig. 2C) and ${ }^{18}$ F-FDG PET/CT (Fig. 2D). No evidence was found of evolution to multiple myeloma. The muscle strength of the legs improved to grade 5 and the patient was able to walk.

\section{Discussion}

SEP is a rare clinical entity of plasma cell neoplasms, particularly when located in the epidural region. Since SEP may be confused with non-Hodgkin's lymphoma, careful histopathological evaluation of the infiltrated cells is crucial for diagnosis. Furthermore, multiple myeloma should be excluded. In this case, histopathology revealed a typical immunopheno- 
type of plasma cells. There were no signs of multiple myeloma following hematological analysis, serum chemistry, serum protein electrophoresis and bone imaging. Thus, a diagnosis of SEP was confirmed.

The optimal management of SEP remains unclear. The generally applied treatment is radiotherapy as SEP is highly radiosensitive. Certain studies report that local radiotherapy may achieve excellent local control (5-6). However, failure to respond is frequent for bulky SEP of $>5.0 \mathrm{~cm}$, which is considered to be a poor prognosis factor for survival $(4-5,7)$. Since in this case the tumor was $>5.0 \mathrm{~cm}$, we treated the patient with bortezomib, based on the fact that bortezomib is effective for relapsed/refractory myeloma, as well as extramedullary relapses of multiple myeloma (1,8-9).

This is the first case of a previously untreated epidural plasmacytoma that was successfully treated with bortezomibcontaining chemotherapy. The combination of bortezomib/ dexamethasone/thalidomide was well tolerated and no side effects were documented. The patients achieved complete and sustained remission. Additionally, we found that MRI is useful in follow-up examinations during therapy, since the images may document a reduction in tumor size. PET/CT scans are also capable of providing additional information to MRI if problems with the MRI technique arise.

In conclusion, this case provides evidence that the use of bortezomib in combination with dexamethasone and thalidomide may be a reliable, safe and effective alternative for treating previously untreated epidural plasmcytomas.

\section{References}

1. Kyle RA and Rajkumar SV: Multiple myeloma. Blood 111: 2962-2972, 2008

2. Okacha N, Chrif E, Brahim E, et al: Extraosseous epidural multiple myeloma presenting with thoracic spine compression. Joint Bone Spine 75: 70-72, 2008.

3. Zazpe I, Caballero C, Cabada T, Guerrero D, Gallo-Ruiz A and Portillo E: Solitary thoracic intradural extramedullary plasmacytoma. Acta Neurochir (Wien) 149: 529-532, 2007.

4. Soutar R, Lucraft H, Jackson G, Reece A, Bird J, Low E and Samson D: Guidelines on the diagnosis and management of solitary plasmacytoma of bone and solitary extramedullary plasmacytoma. Br J Haematol 124: 717-726, 2004.

5. Tsang RW, Gospodarowicz MK, Pintilie M, Bezjak A, Wells W, Hodgson D and Stewart A: Solitary plasmacytoma treated with radiotherapy: impact of tumor size on outcome. Int J Radiat Oncol Biol Phys 50: 113-120, 2001.

6. Galieni P, Cavo M, Pulsoni A, et al: Clinical outcome of extramedullary plasmacytoma. Haematologica 85: 47-51, 2000.

7. Holland J, Trenkner DA, Wasserman TH and Fineberg B: Plasmacytoma. Treatment results and conversion to myeloma. Cancer 69: 1513-1517, 1992.

8. Espanol I, Martinez A, Lopez MD, et al: First report of a medullar cord compression secondary to osseous plasmacytoma successfully treated with bortezomib and dexamethasone. Leuk Res 34: E97-E99, 2010.

9. Laura R, Cibeira MT, Uriburu C, Yantorno S, Salamero O, Blade J and Montserrat E: Bortezomib: an effective agent in extramedullary disease in multiple myeloma. Eur J Haematol 76: 405-408, 2006. 